\title{
EDITORIAL
}

\section{El balance entre producir y usar el conocimiento}

\section{Franklyn Edwin Prieto}

Director de Vigilancia y Análisis del Riesgo en Salud Pública. Instituto Nacional de Salud, Bogotá, Colombia Correspondencia: Dirección: Av Calle 26 N 51-20 Bogotá, Colombia. Tel: 2207700

Correo electrónico: franklyn.prieto@gmail.com

DOI: http://dx.doi.org/10.24267/23897325.222

El mercado de las acciones funciona con un altísimo acceso a la información sobre los movimientos de venta y compra en las bolsas de valores, que permiten tomar decisiones que oscilan entre el bajo y el muy alto riesgo, y que pueden llevar a la riqueza o a la bancarrota a las personas a quienes les administran sus fondos.

La suscripción a los programas de fidelización en las grandes superficies o supermercados proporciona información para que las estrategias de publicidad y mercadeo sean diseñadas de acuerdo con las necesidades de los clientes, al reconocer qué es lo que compran mensualmente y sus variaciones. Los reportes de los movimientos bancarios y crediticios también facilitan reconocer sus posibles ingresos y ubicar en qué situación, ante una administración de impuestos, se puede estar.

Incluso, en nuestras decisiones diarias, utilizamos la información de nuestros recuerdos para establecer si, de acuerdo con las características del ambiente, cargamos sombrilla o no (1); también, si es mejor o no tomar una ruta específica para transportarnos o para sentirnos seguros, a pesar de la existencia de las nuevas aplicaciones de posición geográfica de los dispositivos móviles, o cuándo es la mejor época para tomar unas vacaciones.

En la investigación en salud, también se reconoce esta posibilidad de usar la información rutinaria, la información disponible, más allá de la consecución de nuevos datos. La revisión sistemática, por ejemplo, más que desarrollar nuevas investigaciones, busca utilizar las investigaciones existentes para llegar a recomendaciones ante las divergencias en los resultados, los tamaños deficientes de muestras 
o la necesidad de consolidar el conocimiento. Este tipo de revisión ha evolucionado a reconocer la revisión de revisiones, conocida como revisión "sombrilla" (2), o la revisión realista (3), que recopila la información de intervenciones complejas. En otras situaciones, la revisión sistemática puede partir de las bases de datos de los diferentes ensayos clínicos, para buscar una aproximación real a los resultados. No obstante, en nuestro medio, el real uso de estas revisiones sistemáticas y su expresión en guías de práctica clínica, son limitados en el abordaje individual o colectivo de nuestros pacientes. Gran parte de la crítica hacia los ensayos clínicos controlados -considerados la prueba reina de la medicina basada en la 'evidencia'- está basada en la creación de un escenario "no natural" de atención que limita el reconocimiento de lo que sucedería con el uso de un medicamento a mediano o largo plazo: sus efectos secundarios, la continuidad del efecto clínico, el real efecto clínico o su mortalidad asociada. Los ensayos clínicos pragmáticos resuelven en parte esta situación, pues incorporan la tríada propuesta de la medicina basada en la 'evidencia' de los resultados científicos, las preferencias del paciente y la experiencia del médico (4). Estos ensayos reconocen los reales escenarios de práctica y el efecto, más que la eficacia, de diferentes intervenciones (5). Algunas de nuestras decisiones en el campo clínico no utilizan ni unas ni otras. Una metodología similar se propone en el ámbito de la salud pública y el desarrollo de programas, la investigación operativa (6), que resuelve preguntas cotidianas de investigación en los ámbitos clínicos y comunitarios.

Por otra parte, ante la evidencia de falta de transparencia en algunos ensayos clínicos, pero, en especial del reconocimiento de otras ópticas para el análisis de resultados, la investigación reproducible aborda la posibilidad de repetir el proceso de generación de conocimiento dada la disponibilidad pública de las bases de datos, el plan de análisis y las consultas generadas. Esta investigación reproducible permite, en algunos casos, identificar la fiabilidad, los ajustes o la realidad de los resultados (7). Parte de dicha reproducibilidad se logra en el uso de la información rutinaria de nuestros pacientes.

Algunas agencias, como la UK Medicines and Healthcare Products Regulatory Agency (MHRA), tienen un registro anonimizado de las personas que son atendidas en el sistema de salud, lo que permitiría reconocer los reales efectos en la salud de los medicamentos en la circunstancia real de prueba (8). La recopilación de nuestra estadística clínica sería una herramienta útil para reconocer lo que está pasando con nuestros pacientes, en el ejercicio real de la práctica clínica. El uso de big data ofrece otras oportunidades, como enlazar la información clínica de las personas (historias, resultados de laboratorios, consumos, servicios), con datos sociodemográficos, económicos, educativos, etc., que 
favorecerían una investigación más amplia y más completa. Este uso facilitaría la generación de alertas en tiempo real sobre los verdaderos efectos y las reacciones adversas del uso de diferentes intervenciones e, incluso, para la gestión programática (9).

En la vigilancia de los eventos de interés en salud pública, también se han desarrollado otras formas de detectar incidentes que pueden afectar a la comunidad. El uso de la descripción de los estados de ánimo en las redes sociales permite detectar un aumento de personas que manifiestan "estar enfermos" en sus perfiles, que pueden ser delimitados en tiempo y lugar. La tendencia de las consultas en meta-buscadores de tipo Google, puede identificar patrones de búsqueda de síntomas que alertarían sobre una situación en salud pública. Esto, sumado a la información que se recolecta en la vigilancia rutinaria y en el uso de la información de las grandes bases de datos, facilitaría el reconocimiento de situaciones que amenazan la salud de la población (10).

Esta enumeración de los desarrollos actuales de la investigación en salud podría indicar que podríamos ir hacia el sobrediagnóstico sin que se incida en la realidad de los problemas. Aunque los métodos de investigación evolucionan, el uso de esta información, de este conocimiento, no tiene la misma velocidad o el mismo desempeño para generar cambios en la población. Es tanta la dificultad que se ha diseñado la investigación 'translacional' (11), cuyo objetivo principal es la oportunidad de utilizar el conocimiento de la investigación básica para el desarrollo de intervenciones en salud, aun en el ámbito individual. Lo mismo se ha intentado para llevar los resultados de la investigación en acción en salud pública a su uso en el diseño de acciones, proyectos y programas (12).

La pregunta esencial es: ¿realmente estamos utilizando la información proporcionada por la investigación científica, sea básica o aplicada? En algunos casos es claro que no tenemos información suficiente, pero en muchos otros, no lo es tanto. Si bien los métodos mencionados ofrecen oportunidades para utilizar la información para generar conocimiento, la necesidad va más allá. En los países de bajos y medianos ingresos, necesitamos convertir ese conocimiento en toma de decisiones que resuelvan la carga de enfermedad actual de nuestros países y que controlen el desangre de los sistemas de salud, por la amplia variabilidad en la toma de decisiones individuales.

Este es el real reto. Primero, reconocer estos otros tipos de investigación, descritos previamente. Segundo, traducir la información a los tomadores de decisiones, a las diferentes partes interesadas, a 
los medios de comunicación, a los pares, a la comunidad, desde el diagnóstico de la situación hasta el nivel de recomendaciones y evidencia sobre intervenciones. Tercero, transferir el conocimiento, que significa adopción, adaptación, gestión y mantenimiento. Cuarto, evaluar esta incorporación, que requiere de métodos como los enumerados anteriormente.

Es un reto, sin duda, y esto nos obliga a repensarnos como investigadores, como docentes y como tutores; además, a rediseñar nuestros currículos, a fomentarlo en los estudiantes y profesionales, a producir mensajes claros. Esta conjunción entre generar y utilizar el conocimiento es el real pilar, si, en verdad, queremos cambiar la situación de nuestra población. Es un reto, pero no es imposible.

\section{REFERENCIAS}

1. Rowntree D. Statistics without tears: A primer for non-mathematicians. London: Penguin; 2000. p196

2. Aromataris $E$, Fernández $R$, Godfrey $C M$, Holly C, Khalil H, Tungpunkom P. Summarizing systematic reviews: Methodological development, conduct and reporting of an umbrella review approach. Int J Evid Based Healthc. 2015;13:132-40.

3. Pawson R, Greenhalgh T, Harvey G, Walshe $K$. Realist review--a new method of systematic review designed for complex policy interventions. J Health Serv Res Policy. 2005;10(Suppl.1):21-34.
4. Sackett DL, Rosenberg WMC, Muir JA, Brian $\mathrm{R}$, Scott W. Evidence based medicine: What it is and what it isn't. BMJ. 1996;312:71.

5. Drazen JM, Harrington DP, McMurray JJV, Ware $\mathrm{JH}$, Woodcock J. The changing face of clinical trials: Pragmatic trials. N Engl J Med. 2016;375:454-63.

6. Naidoo $P$, Smuts $B$, Claassens $M$, Rusen ID, Enarson DA, Beyers N. Investigación operativa para mejorar los servicios de salud: una guía para la elaboración de la propuesta. The Union International; 2013. p 96

7. Peng RD. Reproducible research in computational science. Science. 2011;334:1226-7. 
8. Medicines and Healthcare Products Regulatory Agency. The General Practice Research Database (GPRD). Further information for patients. Fecha de consulta: 5 de julio de 2017. Disponible en: http://www.erskinepractice.scot.nhs.uk/website/S11486/files/ GPRD_PatientLeaflet.pdf.

9. European Comission. Study on big data in public health, telemedicine and healthcare. Brussels: European Union; 2016. p 114. Doi: $10.2875 / 734795$

10. Eggleston EM, Weitzman ER. Innovative uses of electronic health records and social media for public health surveillance. Curr Diab Rep. 2014;14:468.
11. Cohrs RJ, Martin T, Ghahramani P, Bidaut L, Higgins PJ, Shahzad A. Translational medicine definition by the European Society for Translational Medicine. New Horizons in Translational Medicine. 2014; 2:86-8.

12. Wilson KM, Brady TJ, Lesesne C. An organizing framework for translation in public health: The knowledge to action framework. Prev Chronic Dis. 2011;8:A46. 